\title{
A NUMERICAL STUDY OF THE EFFECT OF SPACED TRIANGULAR SURFACE WAVES ON NATURAL CONVECTIVE HEAT TRANSFER FROM AN UPWARD FACING HEATED HORIZONTAL ISOTHERMAL SURFACE
}

\author{
Patrick H. Oosthuizen ${ }^{1 *}$, Abdulrahim Kalendar ${ }^{2}$ \\ ${ }^{1}$ Queen's University, Kingston, Ontario Canada K7L 3N6 \\ ${ }^{2}$ College of Tech. Studies, Public Authority for Applied Education and Training, Shuwaikh-24758, Kuwait
}

\begin{abstract}
The use of surface waviness to increase the natural convective heat transfer rate from horizontal surfaces has been extensively investigated and the use of waves with a triangular cross-sectional shape has received significant attention. These studies of the use of triangular shaped waves indicated that the waves do not always produce as significant an increase in the heat transfer rate as anticipated. Most existing studies of the effect of triangular surface waves on the heat transfer rate considered arrangements involving continuous waves, i.e., each wave started where the preceding wave ended However, it may be possible that triangular surface waves would be more effective in increasing the heat transfer rate if they were spaced, i.e., if there was a flat section between each pair of waves. This has been numerically investigated in the present study. Natural convective heat transfer from a horizontal upward facing isothermal heated surface imbedded in a surrounding adiabatic surface has been considered. The heated surface was covered with a series of parallel triangular surface waves running longitudinally along the surface with a space between each pair of waves. Conditions under which laminar, transitional, and turbulent flows exist have been considered. The solution has been obtained using ANSYS FLUENT ${ }^{\odot}$. Results have been obtained for a Prandtl number of 0.74 . The results indicate that using spaced waves produces a higher heat transfer rate than exists with non-spaced waves.
\end{abstract}

KEY WORDS: Natural convection, Horizontal wavy surface, Triangular waves, Spaced waves, Numerical

\section{INTRODUCTION}

The use of surface waviness to increase the natural convective heat transfer from horizontal surfaces has been quite extensively investigated in the past, e.g., see [1-7], and the use of waves with a triangular crosssectional shape in this configuration has received quite significant attention. However, the results of the studies of the use of triangular shaped waves indicate that under some conditions such a wave shape does not produce as great an increase in the heat transfer rate as would be anticipated. Essentially all existing studies of the effect of triangular surface waves on the heat transfer rate considered waves that were continuous, i.e., each wave started where the preceding wave ended. It may be possible that the triangular surface waves would be more effective in increasing the heat transfer rate if they were spaced, i.e., if there was a non-wavy flat section between each pair of waves (see Fig. 1). This possibility has been investigated in the present numerical study. Natural convective heat transfer from a horizontal upward facing isothermal heated surface imbedded in a large surrounding flat adiabatic surface has been considered (see Fig. 2). The heated surface was covered with a series of triangular shaped surface waves running longitudinally along the surface, there

*Corresponding Author: patrick.oosthuizen@queensu.ca 
being, in general, a space between each pair of waves. The parameters defining the wave shape are those shown in Fig. 3. There were two limiting situations: one where there was no space between the surface waves and one where the waves were very thin. These two situations are shown in Fig. 4. Conditions under which laminar flow, transitional flow, and turbulent flow exist have been considered.

This study is part of a broad on-going series of studies of natural convective heat transfer from horizontal heated surfaces, e.g., see [8-12].
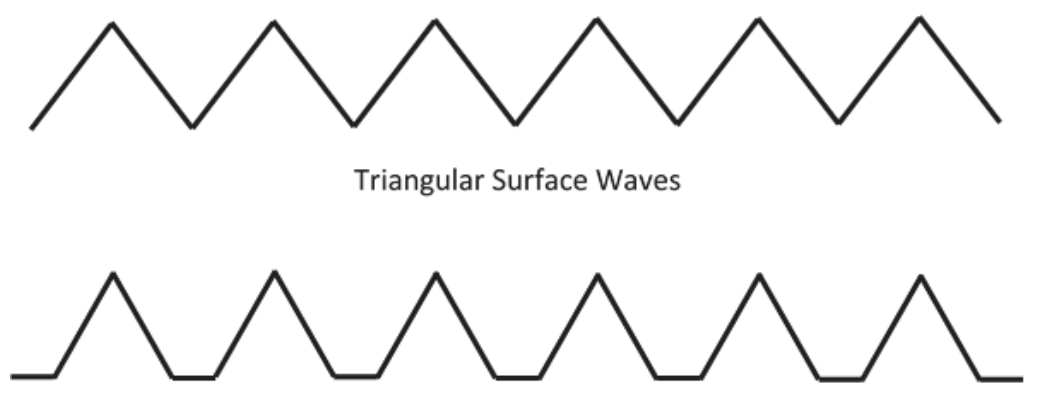

Spaced Triangular Surface Waves

Fig. 1 Continuous, i.e., non-spaced, triangular surface waves (top) and spaced surface waves (bottom).

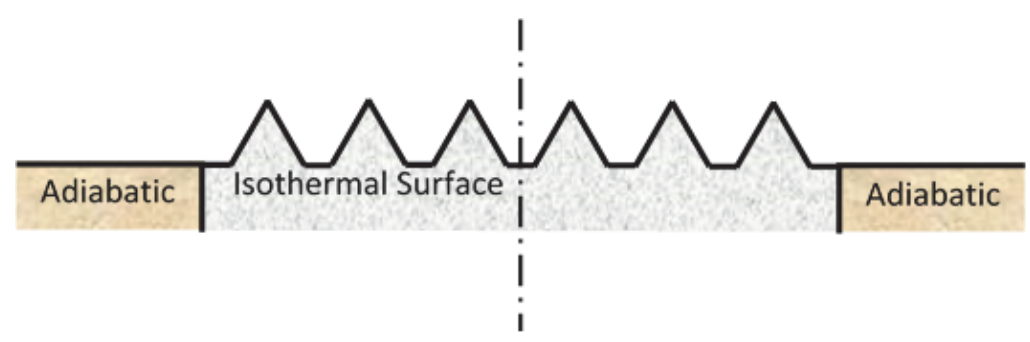

Fig. 2 System considered in the present study.

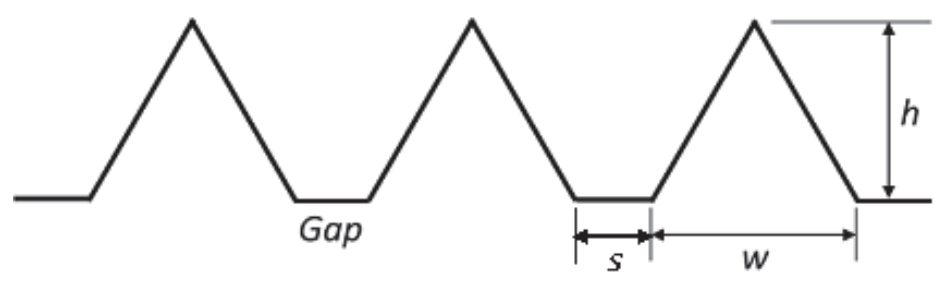

Fig. 3 Geometrical parameters used in defining wave geometry. 


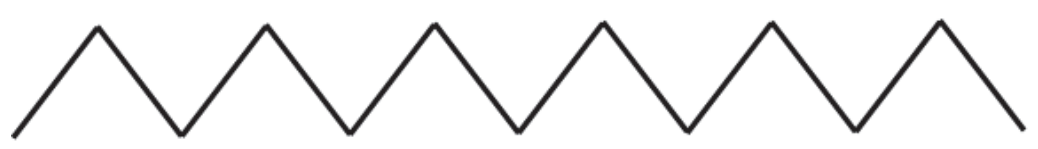

No gap between waves, $W=0.1$

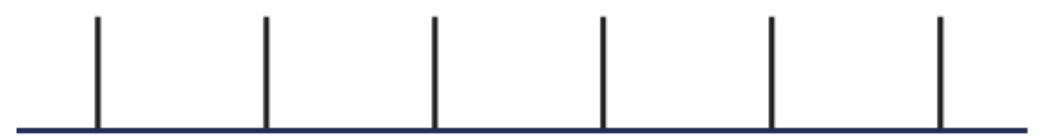

Waves have no thickness, $W=0$

Fig. 4 Limiting cases where there is gap between the waves (top) and where the wave width is very small, i.e., where waves essentially have zero width (bottom).

\section{SOLUTION PROCEDURE}

The flow has been assumed to be steady, two-dimensional, and symmetrical about the centerline through the heated surface shown in Fig. 2. The heated wavy surface has been assumed to be at a uniform temperature, i.e., it has been assumed to be isothermal. The Boussinesq approximation has been used, i.e., the fluid properties have been assumed to be constant except for the density change with temperature that gives rise to the buoyancy forces which is assumed to be linearly dependent on the temperature change. Radiant heat transfer effects have been neglected. The possibility that turbulent flow can develop in the system has been addressed by using the standard $k$-epsilon turbulence model with standard wall functions and with account being taken of buoyancy force effects. The commercial CFD solver ANSYS FLUENT ${ }^{\bigcirc}$ was used to numerically solve the governing equations subject to the boundary conditions. Extensive grid independence and convergence-criteria independence testing was done. To numerically allow for the development of turbulence, the Reynolds averaged governing equations were solved together with a turbulence model for all of the conditions considered. This approach has been used quite extensively in the study of forced convective flows and to some extent in situations involving natural convective flow [13-18]. The results obtained in these studies indicate that while $k$-epsilon turbulence models do not give good predictions of when transition begins in all flow situations, they do appear to provide results of acceptable accuracy for the type of flow situation being considered here.

\section{RESULTS}

The solution has the following parameters:

1. the Rayleigh number, $R a$, based on the overall width, $l$, of the heated surface and the difference between the uniform heated surface temperature, $T_{\mathrm{w}}$, and the temperature of the undisturbed fluid well away from the system, $T_{\mathrm{f}}$, i.e.:

$$
R a=\frac{\beta g l^{3}\left(T_{w}-T_{f}\right)}{v \alpha}
$$

Rayleigh numbers of between approximately $10^{5}$ and $10^{14}$ have been considered in the present study.

2. the number of waves, $n$, which determines the dimensionless distance between the peaks of adjacent triangular waves $W+S=(w+s) / l$. Therefore, since $w+s=l / n$ it follows that:

$$
W+S=\frac{1}{n}
$$


Results have only been obtained for the case where there are ten waves and for this case, $W+S=$ 0.1 . It should be noted that, as shown in Fig. 4, there are two limiting situations: one in which $S=0$ and $W=0.1$ and there is no space between the surface waves; and one in which $S=0.1$ and $W=0$.

3. the value of $W$ which can then be used to find the value of $S$ using $S=0.1-W$.

4. the dimensionless wave height, $H=h / l$.

5. the Prandtl number, Pr. Results have only been obtained for a Prandtl number of 0.74 , which is effectively the value for air at standard ambient conditions.

The heat transfer rates have been expressed in terms of a Nusselt number, $N u$, based on the width, $l$, of the heated surface and on the difference between the temperature of the heated surface, $T_{w}$, and the temperature of the undisturbed fluid that is well away from the system, $T_{f}$, i.e.:

$$
N u=\frac{\overline{Q^{\prime} l}}{A k\left(T_{w}-T_{f}\right)}
$$

where $\overline{Q^{\prime}}$ is the mean heat transfer rate from the heated surface and $A$ is the base area of the heated surface i.e. is the flat area across the bottom of the surface waves. If a unit depth of the surface is considered, the base area is given by $A=l \times 1$.

Since constant values of $n$ and $\operatorname{Pr}$ have been used in obtaining the results presented here it follows that:

$$
N u=\text { function }(R a, W, H)
$$

Typical variations of the Nusselt number, $N u$, and of the Nusselt number for a smooth non-wavy surface with the Rayleigh number, $R a$, for the case where the dimensionless wave height, $H$, is 0.05 for various values of the dimensionless wave width, $W$, are shown in Figs. 5 to 8. Figs. 5 and 8 give the results for the two limiting cases of $W=0.1$ and $W=0$ respectively.

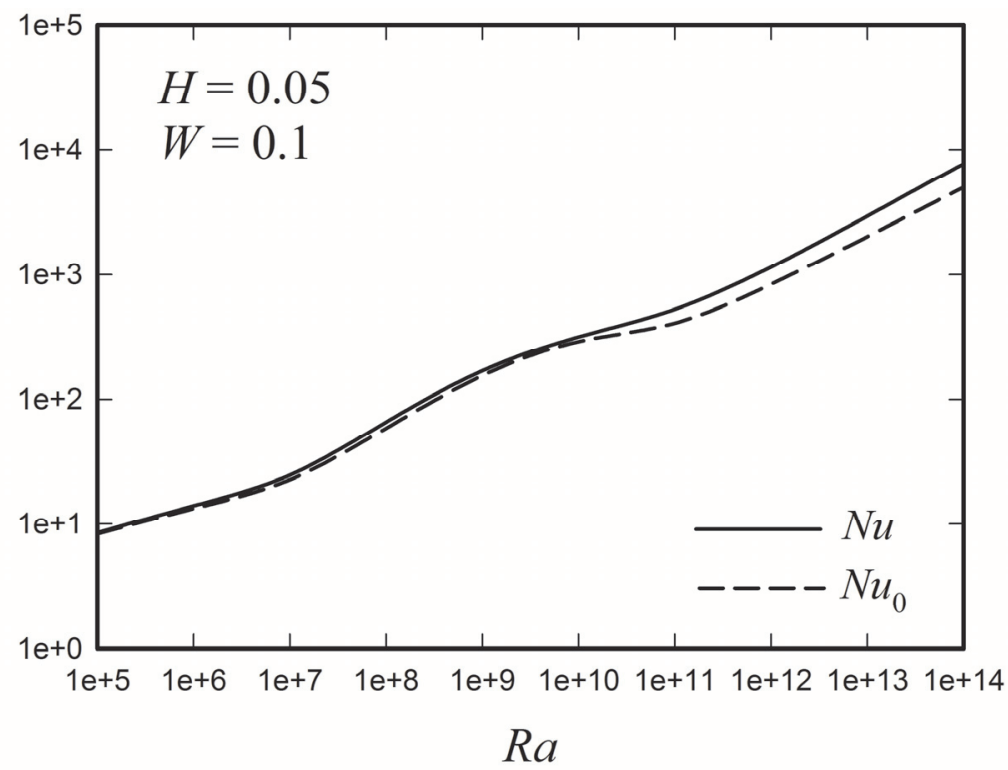

Fig. 5 Variations of the Nusselt number with Rayleigh number for the case of surface waves with a dimensionless height of 0.05 and a dimensionless width of 0.1 (no space between waves) and for the case of a non-wavy surface, i.e., a surface without waves. 
TFEC-2020-32195

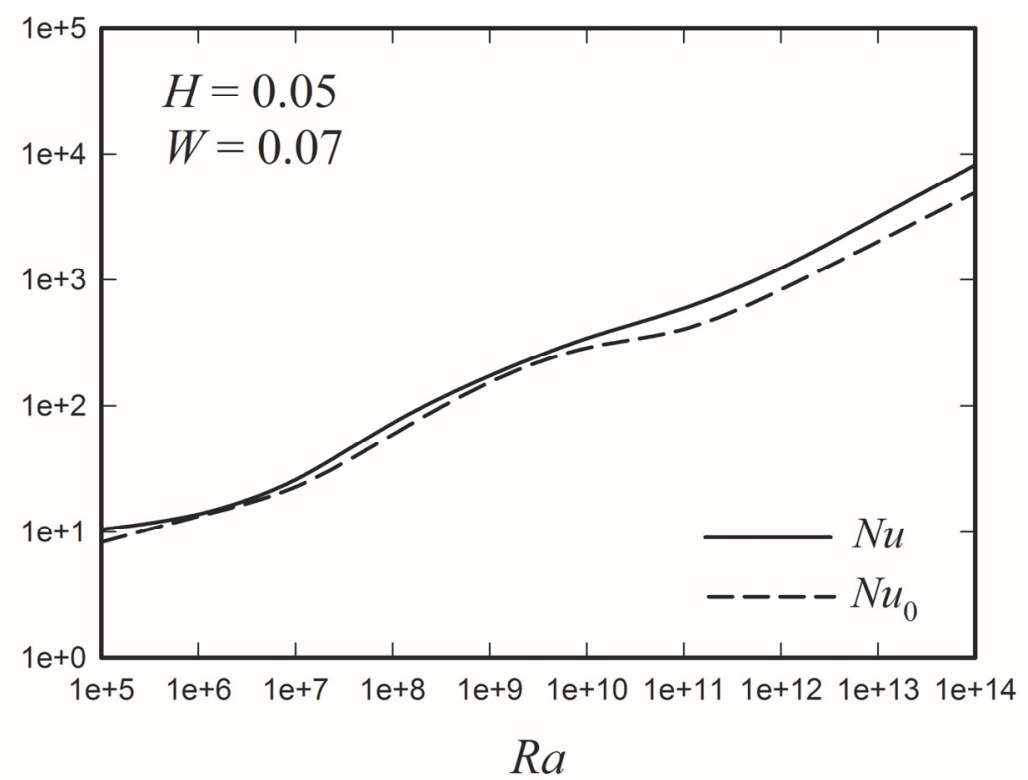

Fig. 6 Variations of the Nusselt number with Rayleigh number for the case of surface waves with a dimensionless height of 0.05 and a dimensionless width of 0.07 and for the case of a non-wavy surface, i.e., a surface without waves.

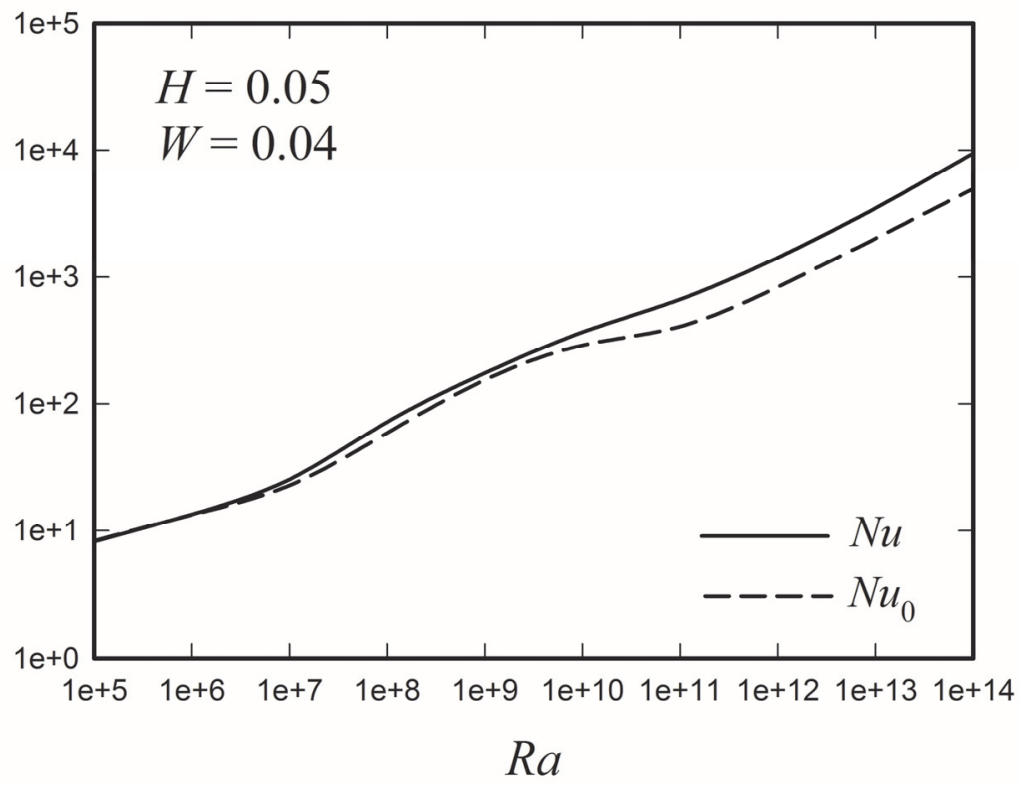

Fig. 7 Variations of the Nusselt number with Rayleigh number for the case where there are surface waves with a dimensionless height of 0.05 and a dimensionless width of 0.04 and for the case of a non-wavy surface, i.e., a surface without waves. 


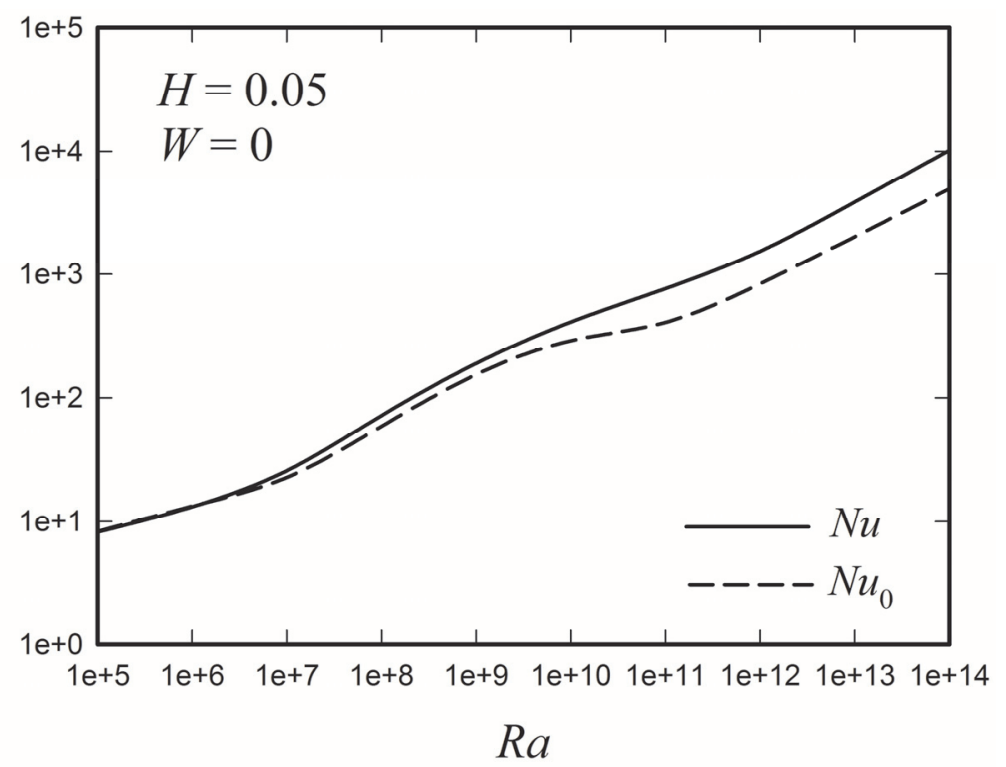

Fig. 8 Variations of the Nusselt number with Rayleigh number for the case where there are surface waves with a dimensionless height of 0.05 and a dimensionless width of 0.04 and for the case of a non-wavy surface, i.e., a surface without waves.

Similar results for the case where the dimensionless wave height, $H$, is 0.03 are shown in Figs. 9 to 12, Figs. 9 and 12 again giving the results for the two limiting cases of $W=0.1$ and $W=0$ respectively.

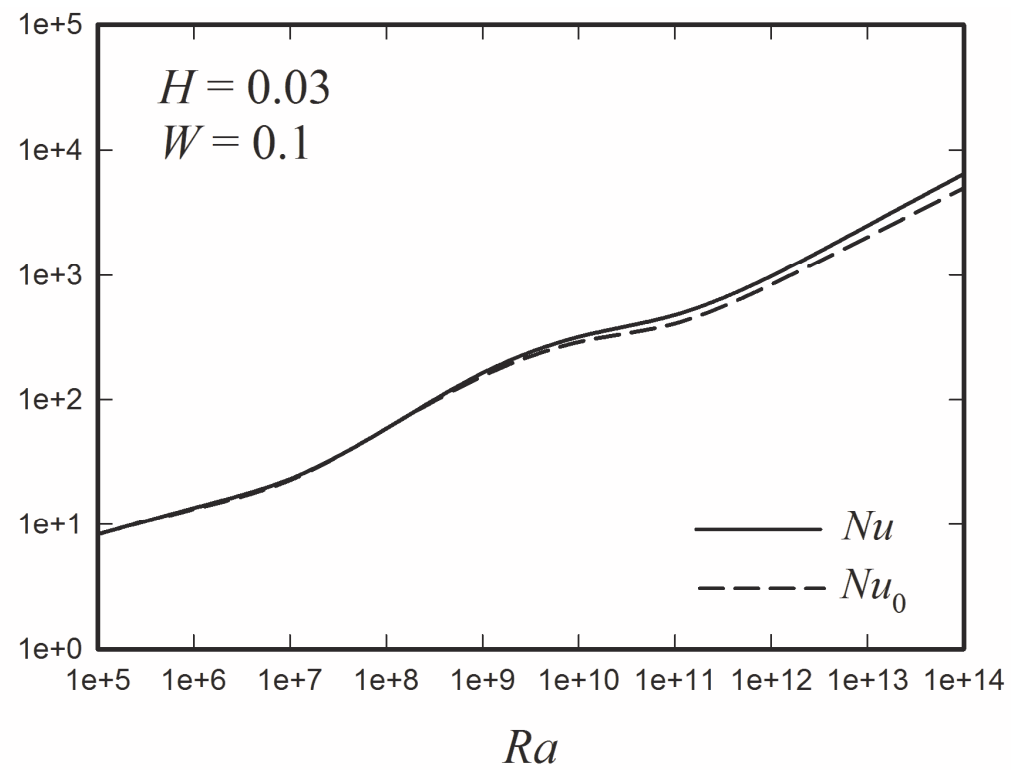

Fig. 9 Variations of the Nusselt number with Rayleigh number for the case where there are surface waves with a dimensionless height of 0.03 and a dimensionless width of 0.1 (no space between waves) and for the case of a non-wavy surface, i.e., a surface without waves. 


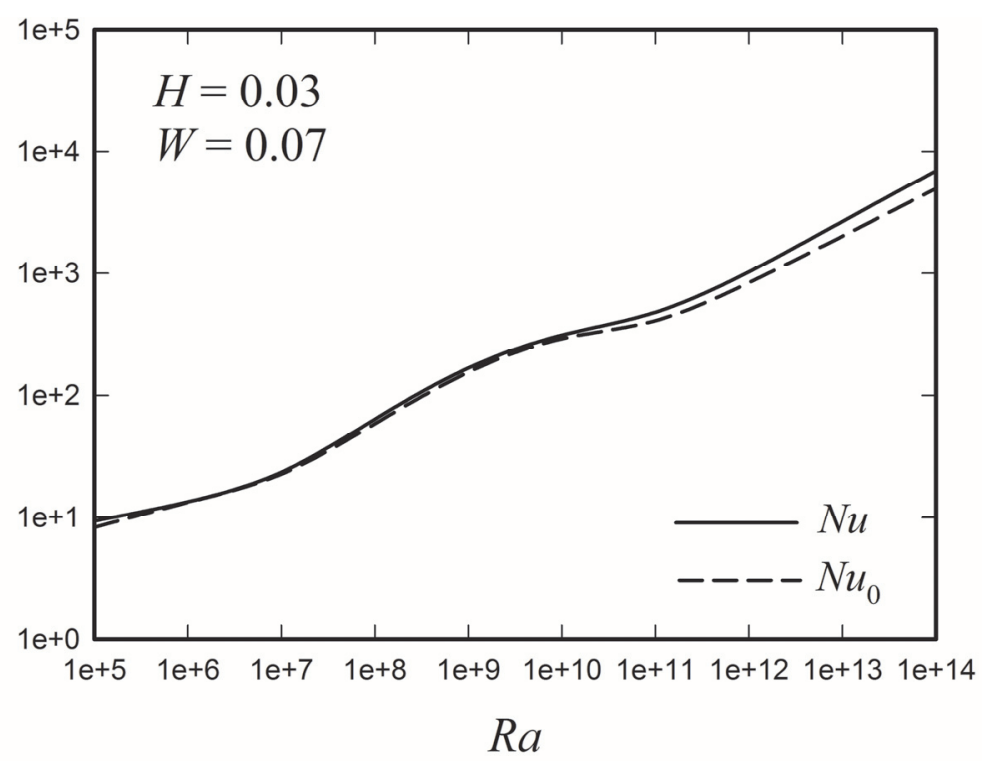

Fig. 10 Variations of the Nusselt number with Rayleigh number for surface waves with a dimensionless height of 0.03 and a dimensionless width of 0.07 and for the case of a non-wavy surface, i.e., a surface without waves.

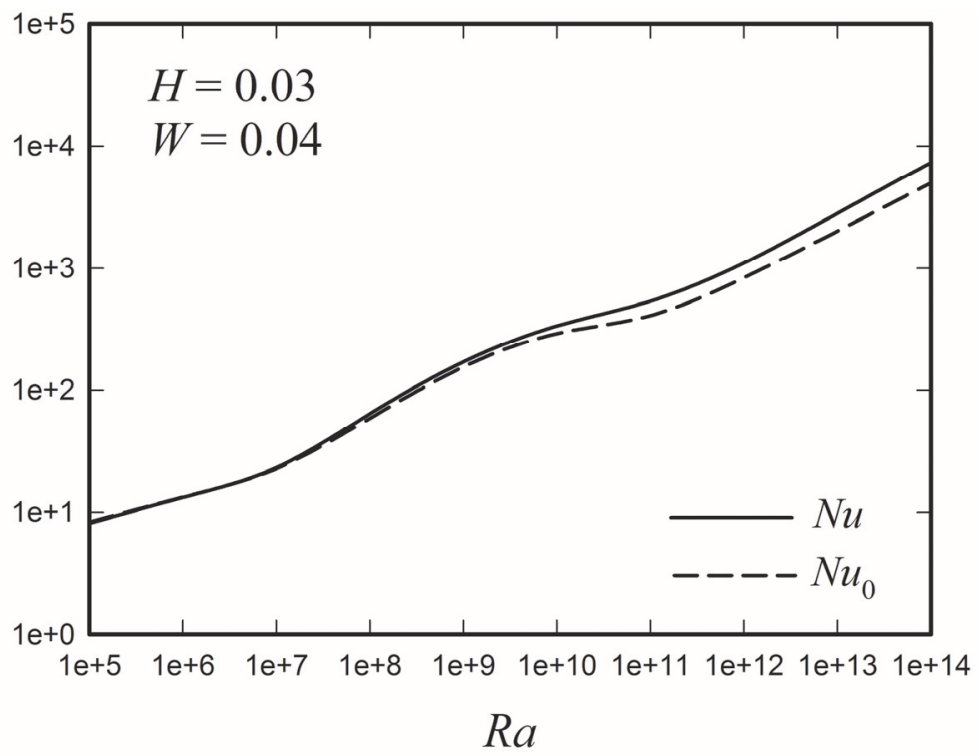

Fig. 11 Variations of the Nusselt number with Rayleigh number for the case where there are surface waves with a dimensionless height of 0.03 and a dimensionless width of 0.04 and for the case of a non-wavy surface, i.e., a surface without waves.

It will be seen from Figs. 5 to 12 that, essentially, in all situations considered the use of the surface waves leads to an increase in the heat transfer rate relative to that which would exist at the same Rayleigh number from a waveless surface. It will also be seen from these figures that the relative increase in the heat transfer rate becomes larger as the Rayleigh number increases and as the dimensionless wave width decreases. This indicates that using spaced waves produces a larger relative increase in the heat transfer rate than exists with continuous, i.e., non-spaced waves. 
TFEC-2020-32195

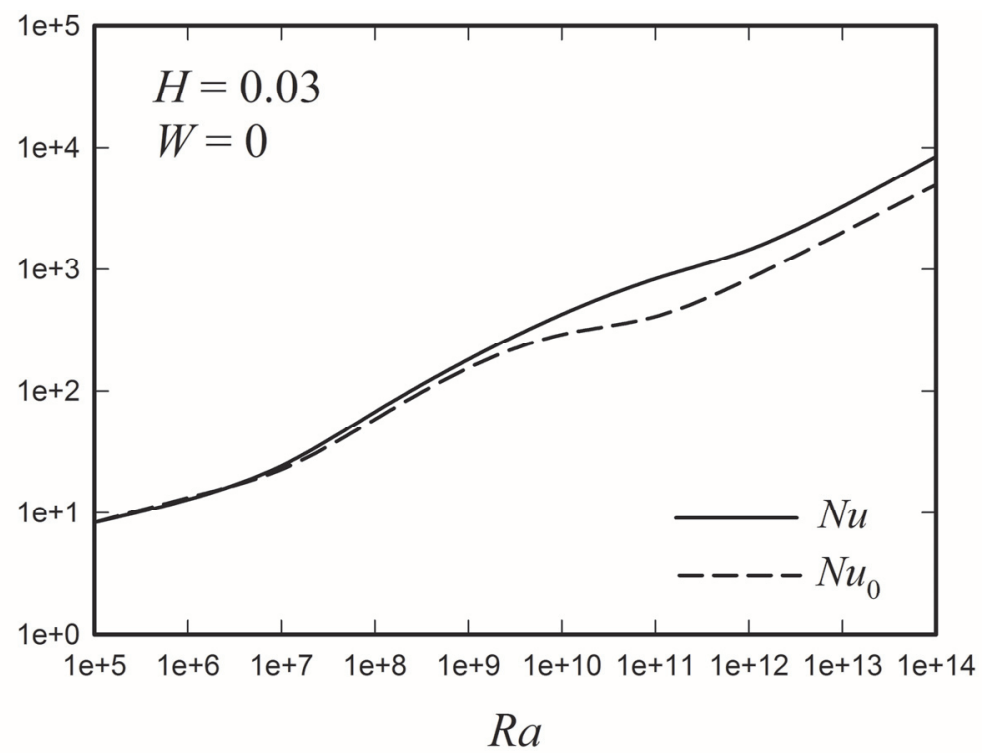

Fig. 12 Variations of the Nusselt number with Rayleigh number for the case where there are surface waves with a dimensionless height of 0.03 and a dimensionless width of 0 and for the case of a non-wavy surface, i.e., a surface without waves.

These conclusions that can be drawn from an examination of Figs. 5 to 12 can be more clearly illustrated by considering the variation with Rayleigh number of the ratio of the Nusselt number to the Nusselt number that would exist at the same Rayleigh number with a non-wavy surface, i.e., a surface without waves. Such variations for $H=0.5$ for $W$ values of 0.1, 0.07, 0.04, and 0 are shown in Figs 13 to 16. Also shown in these figures is the ratio of the actual surface area of the wavy surface to the base area, i.e., the surface area that would exist with a smooth, non-wavy surface.

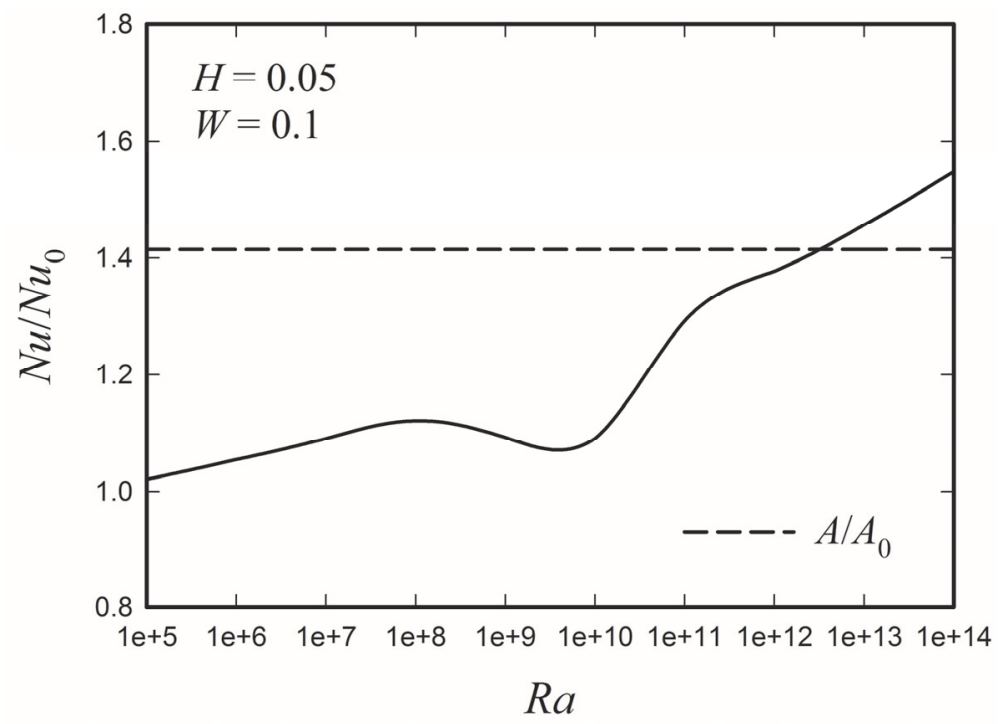

Fig. 13 Variation with Rayleigh number of the ratio of the Nusselt number to the Nusselt number that would exist at the same Rayleigh number with a non-wavy surface, i.e., a surface without waves, for surface waves having a dimensionless height of 0.05 and a dimensionless width of 0.1 . Also shown is the ratio of the actual surface area to the base surface area, i.e., the surface area that would exist with a wave-less surface. 
TFEC-2020-32195

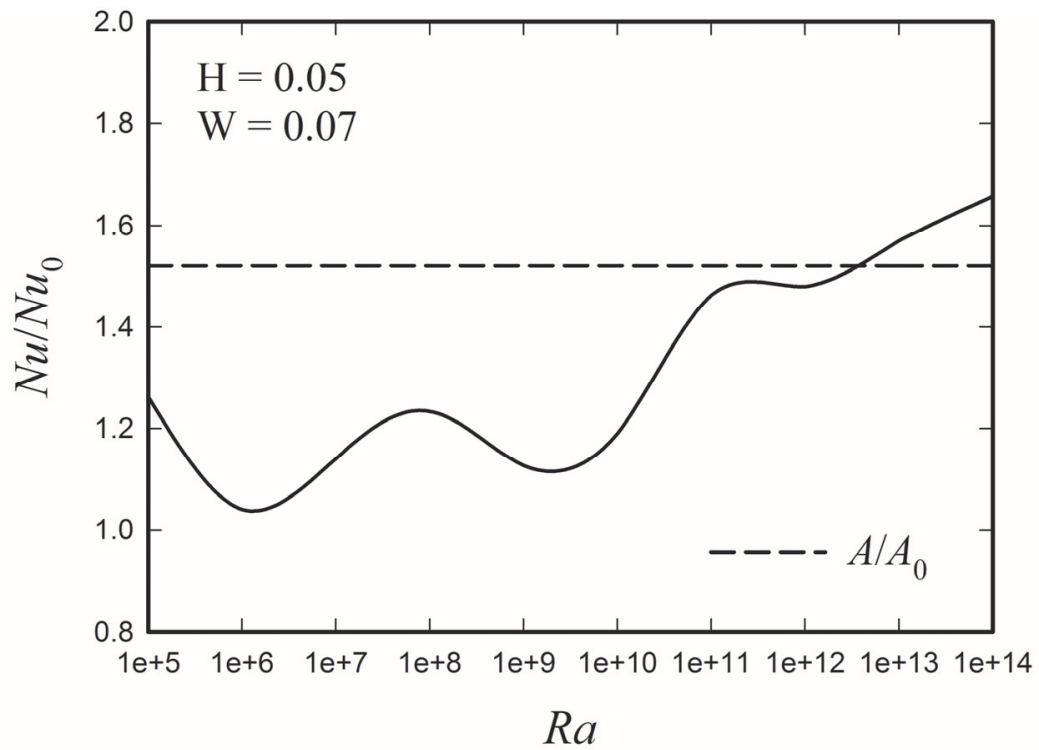

Fig. 14 Variation with Rayleigh number of the ratio of the Nusselt number to the Nusselt number that would exist at the same Rayleigh number with a non-wavy surface, i.e., a surface without waves, for the case where the surface waves have a dimensionless height of 0.05 and a dimensionless width of 0.07 . Also shown is the ratio of the actual surface area to the base surface area, i.e., the surface area that would exist with a wave-less surface.

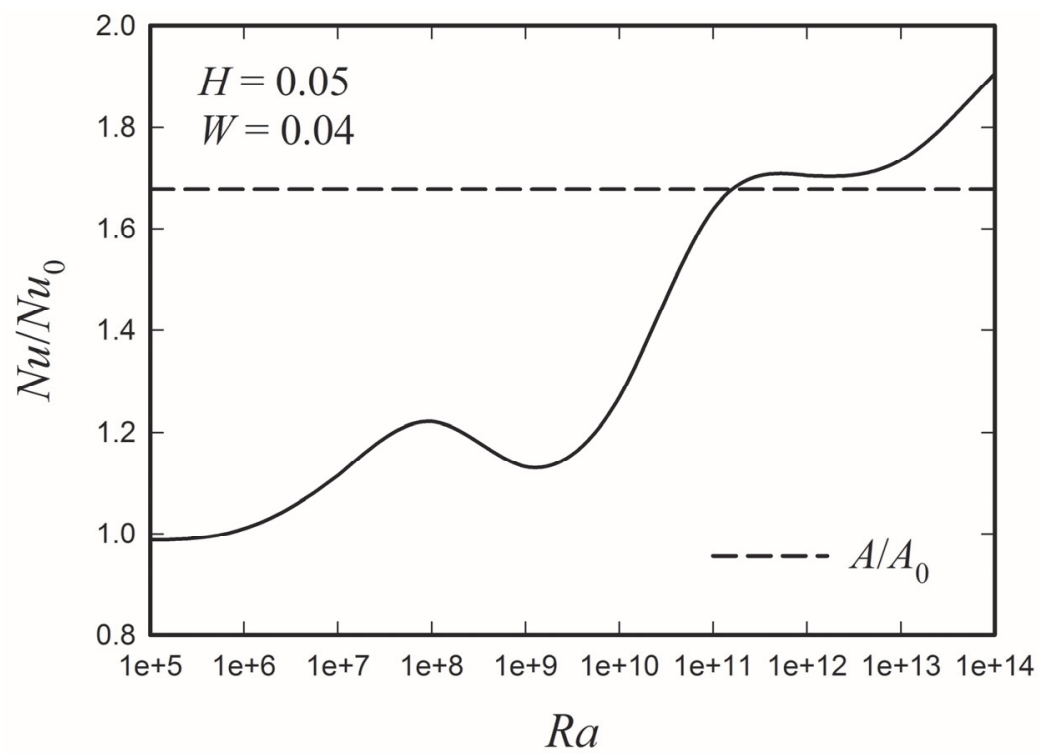

Fig. 15 Variation with Rayleigh number of the ratio of the Nusselt number to the Nusselt number that would exist at the same Rayleigh number with a non-wavy surface, i.e., a surface without waves, for the case where the surface waves have a dimensionless height of 0.05 and a dimensionless width of 0.04 . Also shown is the ratio of the actual surface area to the base surface area, i.e., the surface area that would exist with a wave-less surface. 


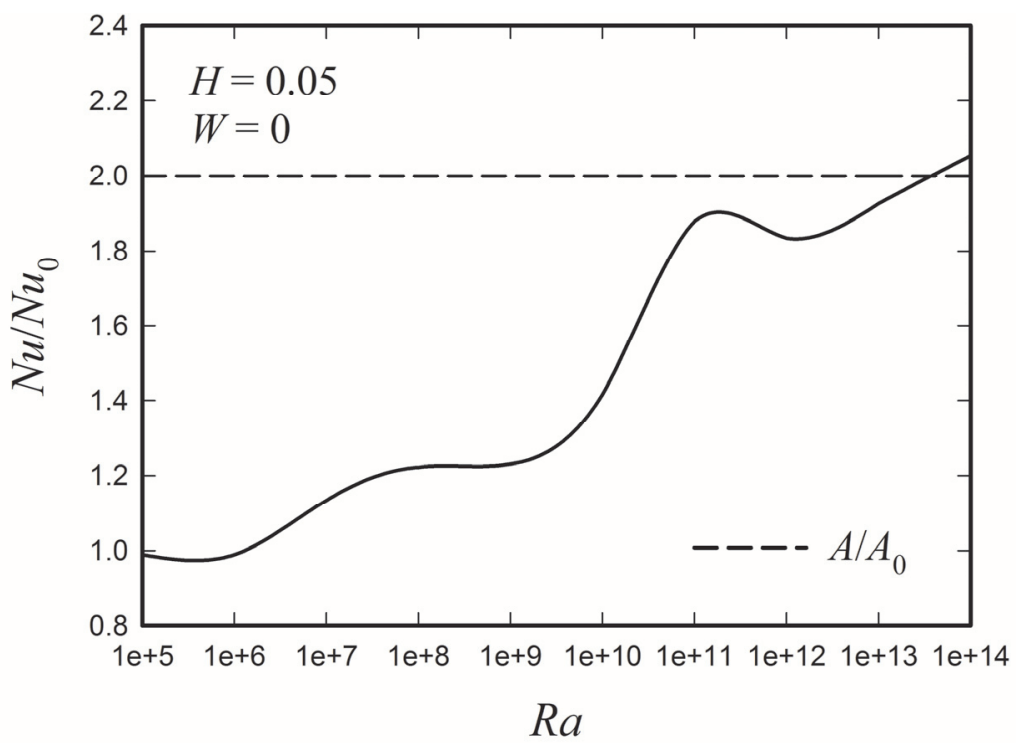

Fig. 16 Variation with Rayleigh number of the ratio of the Nusselt number to the Nusselt number that would exist at the same Rayleigh number with a non-wavy surface, i.e., a surface without waves, for the case where the surface waves have a dimensionless height of 0.05 and a dimensionless width of 0 . Also shown is the ratio of the actual surface area to the base surface area, i.e., the surface area that would exist with a wave-less surface.

Similar results for the case where $H=0.3$ for the same $W$ values are shown in Figs. 17 to 20 .

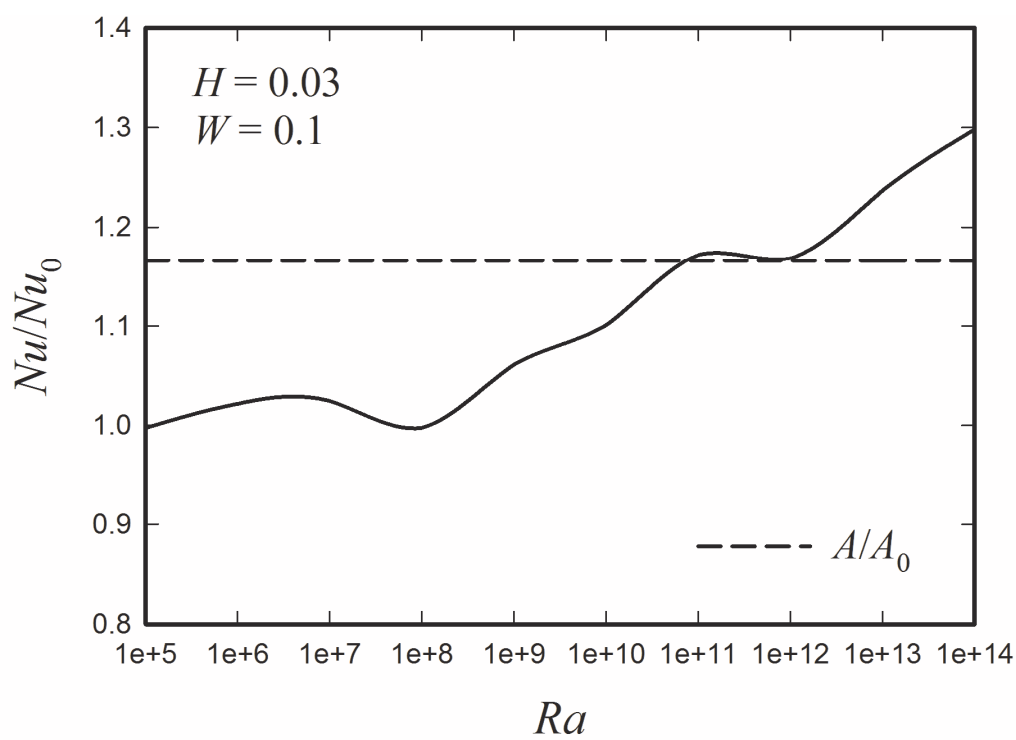

Fig. 17 Variation with Rayleigh number of the ratio of the Nusselt number to the Nusselt number that would exist at the same Rayleigh number with a non-wavy surface, i.e., a surface without waves, for the case where the surface waves have a dimensionless height of 0.03 and a dimensionless width of 0.1 . Also shown

is the ratio of the actual surface area to the base surface area, i.e., the surface area that would exist with a wave-less surface. 


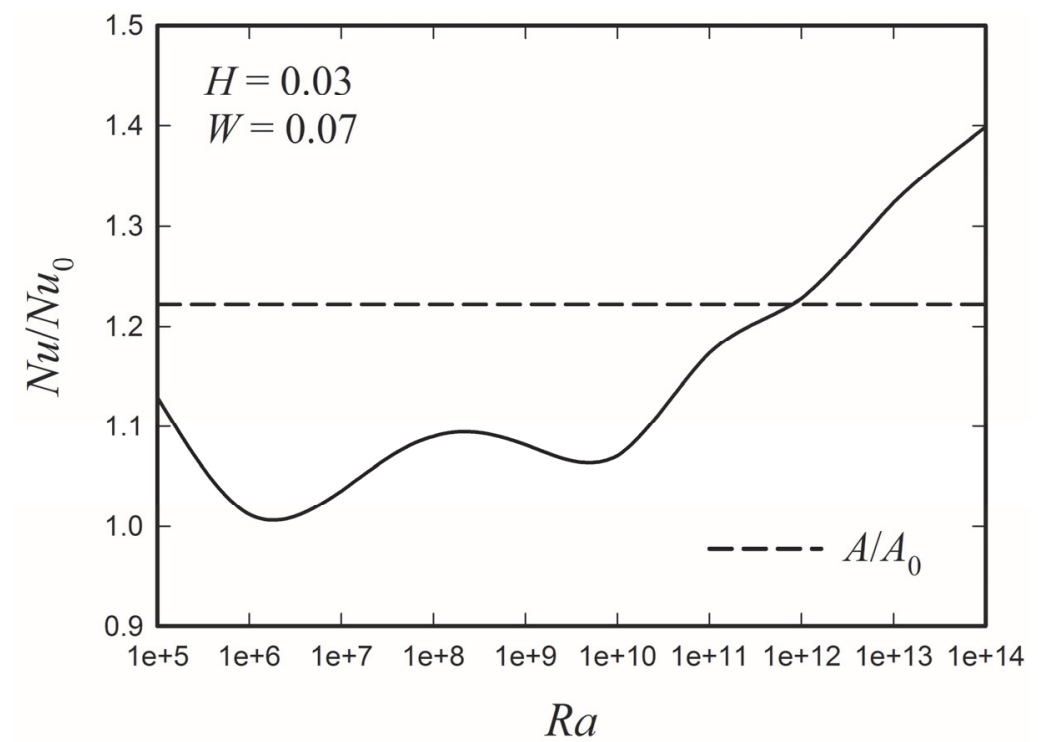

Fig. 18 Variation with Rayleigh number of the ratio of the Nusselt number to the Nusselt number that would exist at the same Rayleigh number with a non-wavy surface, i.e., a surface without waves, for the case where the surface waves have a dimensionless height of 0.03 and a dimensionless width of 0.07 . Also shown is the ratio of the actual surface area to the base surface area, i.e., the surface area that would exist with a wave-less surface.

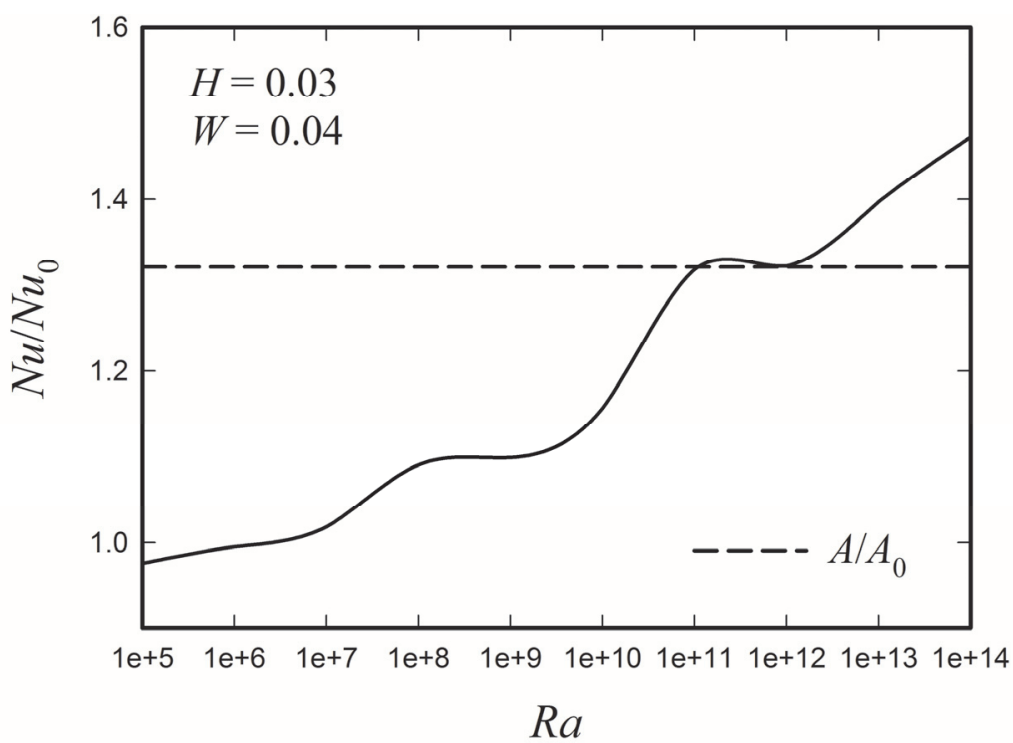

Fig. 19 Variation with Rayleigh number of the ratio of the Nusselt number to the Nusselt number that would exist at the same Rayleigh number with a non-wavy surface, i.e., a surface without waves, for the case where the surface waves have a dimensionless height of 0.03 and a dimensionless width of 0.04 . Also shown is the ratio of the actual surface area to the base surface area, i.e., the surface area that would exist with a wave-less surface. 


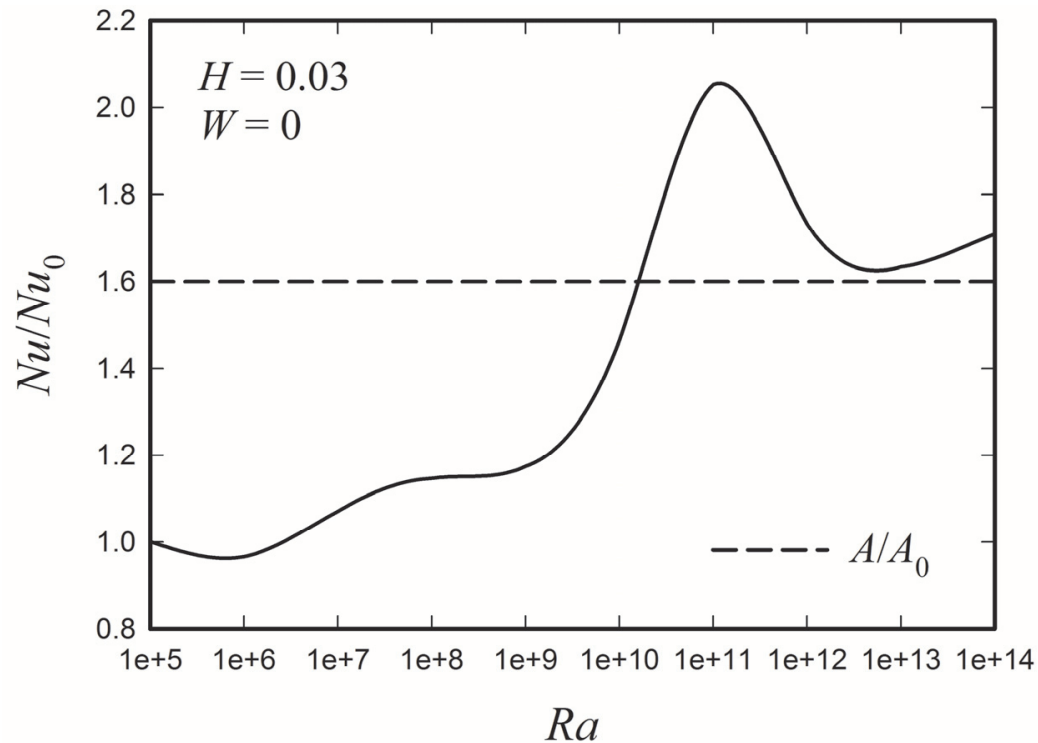

Fig. 20 Variation with Rayleigh number of the ratio of the Nusselt number to the Nusselt number that would exist at the same Rayleigh number with a non-wavy surface, i.e., a surface without waves, for the case where the surface waves have a dimensionless height of 0.03 and a dimensionless width of 0 . Also shown is the ratio of the actual surface area to the base surface area, i.e., the surface area that would exist with a wave-less surface.

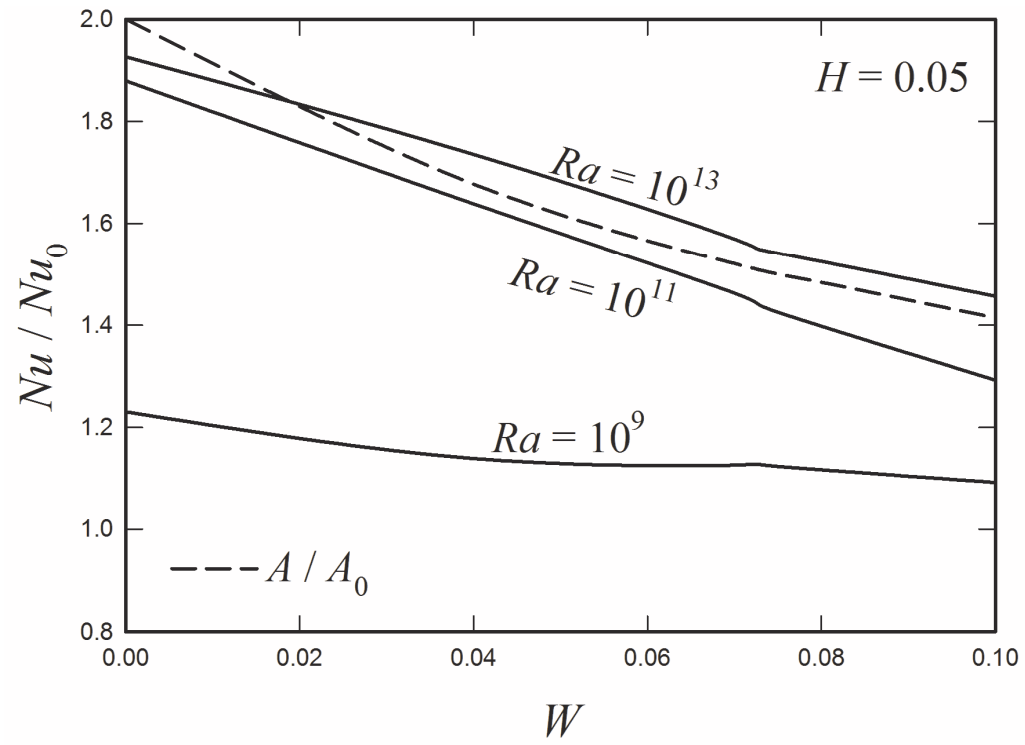

Fig. 21 Variations with dimensionless width of the ratio of the Nusselt number to the Nusselt number that would exist at the same Rayleigh number with a non-wavy surface, i.e., a surface without waves, for various Rayleigh numbers for the case where the surface waves have a dimensionless height of 0.05 Also shown is the variation of the ratio of the actual surface area to the base surface area, i.e., the surface area that would exist with a wave-less surface. 
It will be seen from Figs. 13 to 20 that it is only at the higher Rayleigh numbers considered that the Nusselt number ratio exceeds the surface area ratio. It will also be seen that the Nusselt number ratios relative to the area ratios are roughly the same in all cases but this means that since the area ratio gets larger as $W$ decreases, i.e., as the relative space between the waves increases, the actual enhancement of the heat transfer rate increases as $W$ decreases. This point is illustrated by the results presented in Fig. 21 which shows variations of the ratio of the Nusselt number to the Nusselt number that would exist at the same Rayleigh number with a non-wavy surface with $W$ for three of the higher values of $R a$ considered and for $H=0.5$. The results given in Fig. 21 clearly show, for the Rayleigh number values considered, how the heat transfer enhancement produced by using a wavy surface increases as the value of $W$ decreases.

\section{CONCLUSIONS}

The results obtained in the present study indicate that in essentially all of the situations considered the use of the surface waves leads to an increase in the heat transfer rate relative to that which would exist at the same Rayleigh number from a wave-less surface. The results also indicate that the relative increase in the heat transfer rate gets larger as the Rayleigh number increases and as the dimensionless wave width decreases. This indicates that using spaced waves produces a larger relative increase in the heat transfer rate than exists when continuous, i.e., non-spaced waves are used.

\section{ACKNOWLEDGEMENT}

This work was supported by the Natural Sciences and Engineering Research Council of Canada (NSERC) through its Discovery Grant Program (RGPIN-2015-06444).

\section{NOMENCLATURE}

\begin{tabular}{|c|c|c|c|c|c|}
\hline$A$ & area of heated surface & $\left(\mathrm{m}^{2}\right)$ & $S$ & dimensionless distance between & \\
\hline$A_{0}$ & base area of heated surface & $\left(\mathrm{m}^{2}\right)$ & & waves, $s / l$ & $(-)$ \\
\hline$g$ & gravitational acceleration & $\left(\mathrm{m}^{2} / \mathrm{s}\right)$ & $s$ & distance between waves & (m) \\
\hline$H$ & dimensionless wave height, $h / l$ & $(-)$ & $T_{\mathrm{f}}$ & temperature of undisturbed fluid & \\
\hline$h$ & height of wave & (m) & & far from surface & $(\mathrm{K})$ \\
\hline$k$ & thermal conductivity & $(\mathrm{W} / \mathrm{mK})$ & $T_{\mathrm{w}}$ & temperature of heated surface & $(\mathrm{K})$ \\
\hline$l$ & width of heated surface & (m) & $w$ & base width of wave & (m) \\
\hline$N u$ & mean Nusselt number & $(-)$ & $W$ & dimensionless wave base width, & \\
\hline$N u_{0}$ & Nusselt for waveless surface & & & $w / l$ & $(-)$ \\
\hline & at same Rayleigh number & $(-)$ & $\alpha$ & thermal diffusivity & $\left(\mathrm{m}^{2} / \mathrm{s}\right)$ \\
\hline$n$ & number of waves on surface & $(-)$ & $\beta$ & bulk coefficient of thermal & \\
\hline $\operatorname{Pr}$ & Prandtl number & $(-)$ & & expansion & $(1 / \mathrm{K})$ \\
\hline$\overline{Q^{\prime}}$ & mean surface heat transfer rate & $(\mathrm{W})$ & $v$ & kinematic viscosity & $\left(\mathrm{m}^{2} / \mathrm{s}\right)$ \\
\hline$R a$ & Rayleigh number based on $l$ & $(-)$ & & & \\
\hline
\end{tabular}

\section{REFERENCES}

[1]. Siddiqa, S., Hossain, M.A., Gorla, R.S.R., "Natural convection flow of viscous fluid over triangular wavy horizontal surface," Computers and Fluids, 106, pp. 130-134, (2015).

[2]. Siddiqa, S., Hossain, M.A., "Natural convection flow over wavy horizontal surface," Advances in Mechanical Engineering, 5, pp. 743034-743040, (2013).

[3]. Siddiqa, S.; Hossain, M.A.; Saha, S.C., "The effect of thermal radiation on the natural convection boundary layer flow over a wavy horizontal surface," International Journal of Thermal Sciences, 84, pp. 143-150, (2014). 
[4]. Pretot, S., Miriel, J., Bailly, Y., Zeghmati, B., "Visualization and simulation of the natural-convection flow above horizontal wavy plates," Numerical Heat Transfer, Part A (Applications), 43(3), pp. 307-25, (2003).

[5]. Pretot, S., Zeghmati, B., Caminat, P., "Influence of surface roughness on natural convection above a horizontal plate," Advances in Eng. Software, 31, pp. 793-801, (2000).

[6]. Oosthuizen, P.H., "A Numerical Study of Natural Convective Heat Transfer from a Horizontal Isothermal Surface with Rectangular Surface Roughness Elements," Proc. of $1^{\text {st }}$ Pacific Rim Thermal Engg. Conf., Paper PRTEC-14630, (2016).

[7]. Oosthuizen, P.H., Paul, J.T., "A Numerical Study of Natural Convective Heat Transfer from an Inclined Isothermal Plate with a 'Wavy' Surface," Proc. of ASME 2011 Int. Mechanical Engg. Congress \& Exposition (IMECE2011), Paper IMECE2011$65010,(2011)$.

[8]. Oosthuizen, P.H., "A Numerical Study of Natural Convective Heat Transfer from a Horizontal Isothermal Square Element Imbedded in an Adiabatic Surface with a Parallel Adiabatic Covering surface," Proc. of $10^{\text {th }}$ Int. Conf. on Heat Transfer, Fluid Mechanics and Thermodynamics, Paper 1569876763, (2014).

[9]. Oosthuizen, P.H., "A Numerical Study of Natural Convective Heat Transfer from a Horizontal Isothermal Square Element with an Unheated Square Adiabatic Inner Section," Proc. of $10^{\text {th }}$ Int. Conf. on Heat Transfer, Fluid Mechanics and Thermodynamics, Paper 570075655, (2015).

[10].Oosthuizen, P.H., "Natural Convective Heat Transfer from a Horizontal Rectangular Isothermal Element Imbedded in a Plane Adiabatic Surface with a Parallel Adiabatic Covering Surface," Proc. of ASME 2014 Int. Mechanical Engg. Congress and Exposition (IMECE2014), Paper IMECE2014-36780, (2004).

[11]. Oosthuizen, P.H., "Laminar and Turbulent Natural Convective Heat Transfer from a Horizontal Rectangular Isothermal Element Imbedded in a Flat Adiabatic Surrounding Surface," Proc. of $6^{\text {th }}$ Int. Symposium on Advances in Computational Heat Transfer, Paper CHT-15-145, (2015).

[12]. Oosthuizen, P.H., "A Numerical Study of Natural Convective Heat Transfer from Horizontal Isothermal Heated Elements of Complex Shape," Proc. of $1^{\text {st }}$ Thermal and Fluids Engg. Summer Conf. (ASTFE), Paper TFESC-12863, (2015).

[13].Savill, A.M., "Evaluating turbulence model predictions of transition. An ERCOFTAC special interest group project," Applied Scientific Research, 51, pp. 555-562, (1993).

[14]. Schmidt, R.C., Patankar, S.V., "Simulating boundary layer transition with low-Reynolds-number $k-\varepsilon$ turbulence models: Part $1-$ an evaluation of prediction characteristics," J. Turbomachinery, 113, pp. 10-17, (1991).

[15].Plumb, O.A., Kennedy, L.A., "Application of a $k$-e turbulence model to natural convection from a vertical isothermal surface," Journal of Heat Transfer, 99, pp. 79-85, (1977).

[16].Zheng, X., Liu, C., Liu, F., Yang, C.-I., "Turbulent transition simulation using the k- $\omega$ model," International Journal for Numerical Methods in Engineering, 42, pp. 907-926, (1998)

[17]. Albets-Chico, X., Oliva, A., Perez-Segarra, C.D., "Numerical experiments in turbulent natural convection using two-equation eddy-viscosity models," Journal of Heat Transfer, 130(7), pp. 072501-1-072401-11, (2008).

[18].Xamán, J., Álvarez, G., Lira, L., Estrada, C., "Numerical study of heat transfer by laminar and turbulent natural convection in tall cavities of façade elements," Energy and Buildings, 37(7), pp. 787-794, (2005). 